\title{
Electrophoresis Profile of Total Peroxidases in Sera and Saliva of Patients with Different Oral Tumors
}

\author{
HATHAMA RAZOOKI HASAN* and NUHA NIHAD A. ABURAHMA* \\ ${ }^{*}$ Department of Chemistry, College of Science, Baghdad University, Iraq. \\ ${ }^{*}$ Corresponding author E-mail: hathamahasan@ scbaghdad.com
}

http://dx.doi.org/10.13005/ojc/300110

(Received: February 12, 2014; Accepted: March 03, 2014)

\begin{abstract}
Total peroxidase system (EC 1.11.1.X) activity is known to play a key role in a number of human diseases, where the activity of these species can be both beneficial \& detrimental. In our previous work (submitted for publication) a remarkable increase have been noticed in the activity of this system in saliva of patients with oral tumors (Oral Squamous Cell Carcinoma OSCC), \& Ossifying Fibroma, OF). The present project aimed to highlight the variations in the different forms of this system in saliva \& serum samples among patients with above mentioned tumors, in comparison to that of corresponding healthy individuals, using the electrophoresis as the analytical tool. Salivary peroxidase gave faint bands with a poor separation when the analysis was carried out using basic PAGE electrohoresis while good clear bands, as well as better resolutions of these bands were obtained when acidic PAGE electrophoresis was used for the analysis. An additional band, moved further toward the anode, was observed to be present, as the electrozymogram indicated, in the saliva samples of the patients with malignant tumors (Squamous cell carcinoma). The results also showed that using benzidine, or o-dianisidine, as the substrate in staining of the polyacrylamide gels, in order to localize the bands that exhibit peroxidase activity, seems to be better than using 3,3 ', 5,5'-Tetramethylbenzidine (TMBZ) as the substrate for this purpose.
\end{abstract}

Key words: Oral squamous cell carcinoma (OSCC), Ossifying Fibroma (OF), Peroxidase system, electrophoresis.

\section{INTRODUCTION}

Peroxidase activity in human body, is found in the exocrine secretions including milk, tears, and saliva as well as in viginal fluid ${ }^{1,2}$. Most of this activity is derived from the enzyme synthesized in the glands that produce these secretions, in addition from different types of white blood cells. Human whole saliva contains two types of peroxidases: salivary peroxidases (SPO) which present in different isoforms, existing as an equilibrium mixture of monomers and aggregates, and this monomer have an alkaline isoelectric point, and a molecular weight of $78-80 \mathrm{KDa}^{3,4}$ and myeloperoxidase (MPO) which is a homodimer cationic protein, with molecular weight $130-160 \mathrm{KDa}^{3,4}$. The latter type enzyme was also reported to be present in different isoforms, among which three of them are the major isoforms ${ }^{5,6}$ and they have been reported to be part of the innate host defence in oral cavity ${ }^{3}$. 
The peroxidases system is known to play a key role in a number of human diseases where the activity of these species can be both beneficial and detrimental. In recent years, there have been a dramatic increase in our understanding of the enzymology, biochemistry, and biological roles of these species, although much remains to be discovered with regard to the nature of the major damaging species generated by these enzymes, the critical targets of these oxidants, and their role in both beneficial reactions and tissue pathology ${ }^{7}$.

Scientists have studied the correlation of the peroxidases enzymes activity with different diseases and the physiological stats where oxidative stress is implicated. The salivary antioxidant system, in which the peroxidase is the pivotal enzyme, has been drawing increased attention in recent years. This enzyme seems to be of paramount importance in the oral defense mechanism, especially against the attack of free radicals related to the evolution of oral cancer ${ }^{8}$.

In our laboratory a remarkable increase in the activity of total peroxidase system (EC 1.11.1.X) has been previously reported in saliva of patients Oral Epithelial Tumors and oral tumors ${ }^{9}$ (oral squamous cell carcinoma (OSCC) and oral ossifying fibroma (OF)).

The main goal of the present work is to highlight the variations in the different forms of this enzyme system, in saliva and sera samples among patients with oral tumors in comparison to that of corresponding healthy individuals using electrophoresis as the analytical tool.

\section{MATERIAL AND METHODS}

\section{Chemicals}

All chemicals used throughout this work were of highly purified grade.

\section{Studied groups}

Patients $(n=18)$ with oral squamous cell carcinoma (OSCC), \& patients $(n=20)$ with Ossifying Fibroma (OF) were included in the present study. These patients were attending the Department of Oral \& Maxillofacial Surgery ( Al- Wasity Hospital
\& Hospital of Specialized Surgeries,Medicallity in Baghdad/Iraq. The control groups were healthy matched gender and age individuals.

\section{Samples}

Pooled samples of unstimulated whole, mixed saliva samples \& sera samples of the two groups of the patients, as well as the control groups were analyzed by electrophoresis. The saliva \& blood samples were collected on ice, under resting conditions in a quiet room between 8.0-9.0 a.m. The patients and the healthy individuals were overnight fasting \& asked to rinse their mouth with normal saline, before giving the saliva samples.

\section{Conventional polyacrylamide gel electrophoresis} (7.5) ${ }^{10}$

In this study, anodic conventional polyacrylamide gel electrophoresis $(7.5 \%)$ was carried out according to LKB 2117 note using trisglycine buffer stock solution $(0.15 \mathrm{M}) \mathrm{pH} 8.9$, for separation of peroxidase.

\section{Cathodic polyacrylamide gels electrophoresis (9\%)}

The method of Azen ${ }^{11}$, was used for the optimal separation of multiple forms of peroxidase in saliva in which citric acid-glycine buffer (0.37M) $\mathrm{pH} 4$ was used as stock solution.

In both anodic and cathodic conventional PAGE a volume of $(10 \mu \mathrm{l})$ of the samples were applied into the wells in the gel, and concentrated for 5-10 min. with a current of $20 \mathrm{~mA}$, electrophoresis was continued using $40 \mathrm{~mA}$, and $15 \mathrm{~V} / \mathrm{cm}$ for( 3.5-4) hours or until the stain dye reached the gel margin.

\section{Gel Staining Methods \\ Peroxidase Activity Staining}

In order to localize the peroxidase activity in the studied saliva, and sera samples, on the polyacrylamide gel, the gels were stained using either of the following staining methods.

\section{For Sera samples}

After the electrophoresis run was finished three different substrates were used and as follows. 


\section{O-dianisidine stain}

The peroxidase activity bands were detected by using O-dianisidine, as a Substrate $12 \& 13$.

\section{Benzidine stain}

The gel was stained for peroxidase activity by using benzidine, as a Substrate and as described by Lee ${ }^{14}$.

\section{3, 3', 5, 5'-Tetramethylbenzidine}

The gels were stained using 3, 3', 5, 5'Tetramethylbenzidine, as the Substrate \& according to Thomas ${ }^{15}$.

\section{For saliva samples}

To detect the presence of peroxidases activity in the saliva samples of the different groups included in the current study, at the end of the electrophoresis run, the gels were stained, as described above in ( 1and 2 ).

\section{RESULTS AND DISCUSSION}

The convential electrophoresis technique which is used as the analytical tool throughout the

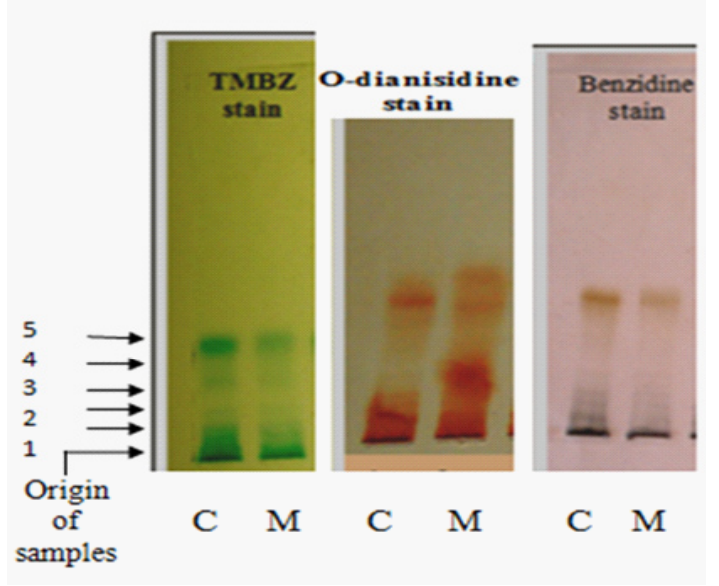

Fig. 1: Electrozymogram showing peroxidases profile. The electrophoresis was carried out using conventional $7.5 \%$ polyacrylamide gel. The gels were stained for peroxidase activity using either of TMPZ, or O-dianisidine, or Benzidine as a substrate. (C) refers to pooled control crude sera \& (M) refers to pooled crud sera of patients with oral malignant tumor (Oral squamous cell carcinoma OSCC) present study, is based on the polymerization of acrylamide with N, N'-methylene-bis-acrylamide (Bis) as the cross-linking agent. This free radical polymerization method, uses ammonium persulphate as the initiator and $\mathrm{N}, \mathrm{N}, \mathrm{N}$ ', N'-tetramethylethylenediamine (TEMED) as the catalyst, the degree of cross-linking can be partly controlled by adjusting the ratio of acrylamide to bis ${ }^{16}$.

The detection of peroxidase different isoforms present in the sera samples of Oral Squamous Cell Carcinoma group and Ossifying Fibroma group included in this work was carried out using three different staining methods, Tetra methyl benzidine (TMBZ) stain, o-dianisidine stain, and Benzidine stain. The results are presented in figures 1 and 2.

Fig. 1 and 2 shows the comparison of separated peroxidase isoforms in sera of the two studied groups, stained with three different staining methods. As it is clear from the electrozymogram, all the used staining methods are suitable for the detection of the peroxidase activity, they gave clear distinct bands. Meanwhile it was noticed that when TMBZ stain was used, the resolution of the bands

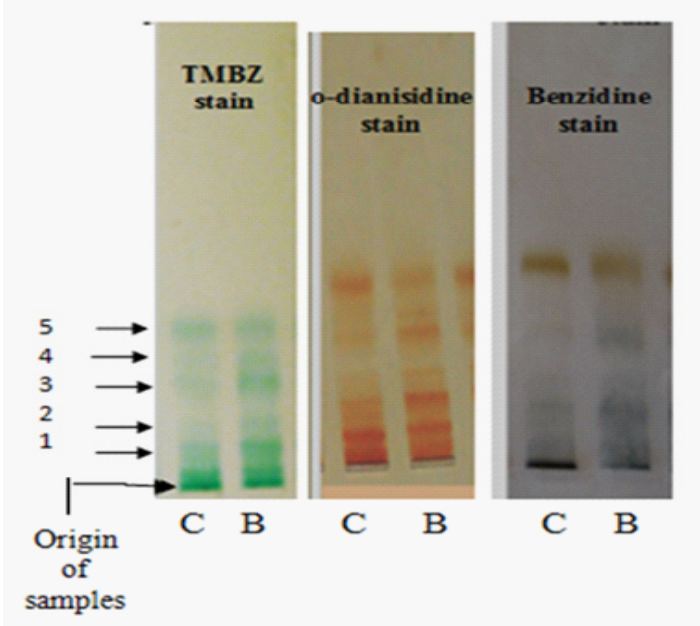

Fig 2: Electrozymogram showing peroxidase profile. The electrophoresis was carried out using convential $7.5 \%$ polyacrylamide gel

.The gels were stained for the peroxidase activity using either of TMPZ, or O-dianisidine

or Benzidine. (C) refers to pooled control crude sera \& $(B)$ refers to pooled crude sera of patients with benign oral tumors (Ossifying Fibroma OF) 
were better, but these bands disappeared quickly. On contrast, with Benzidine stain and o-dianisidine stain, both produced less resolution than TMBZ stain, but the bands were stable by time. It is also clear from Fig. 1 and 2 that different forms of the enzyme are present in the sera of the two studied groups. These enzyme forms differ in their relative mobilities, which reflect changes in their molecular weights and charges. In addition to this, it is obvious from the comparison of the peroxidase separation pattern, that the enzyme in the sera of the control group present in five distinct forms. It was also observed from the electrozymogram (Fig. 1 ) that the peroxidase activity bands show a decrease in their intensity in the malignant group oscc when compared with that of the control group particularly in bands 3, 4 and 5. As far as the enzyme in the second group (OF) is concerned (Fig. 2) the same peroxidase numbers of bands were observed to be present in the sera of these patients, with also an alteration in their intensity when compared to their corresponding control group.

The peroxidase enzyme has been reported to have a wide range of biological functions, and takes parts in either antibacterial action, or cellular defense against oxidative damage. The overproduction of hydrogen peroxide which formed as results of both dismutation of superoxide radicals by superoxide dismutase, and immune cells leads to increased peroxidase activity ${ }^{3}$.

The Peroxidases were reported to be present in human whole saliva, in two different types: -the first one with basic and the second with acidic isoelectric point ${ }^{16}$, so in the current study anodic $(7.5 \%)$, and cathodic (9\%) conventional PAGE , were used to highlight the differences in peroxidase forms which present in the saliva of the studied groups. When the convential electrophoresis PAGE was used, the polymerization of the acrylamide gel was done using ammonium persulphate \& TEMED. While the polymerization of the acidic gel was done by ferrous sulfate, ascorbic acid, and hydrogen peroxide.

Since when the TMBZ was used as the substrate in the staining procedure of the gel for the peroxidase activity, the visualized peroxidase activity bands disappeared quickly, the peroxidases activity in the saliva samples of the studied groups was detected by using either O-dianisidine, or Benzidine. The obtained electrophoresis patterns are presented in Figures 3 and 4.

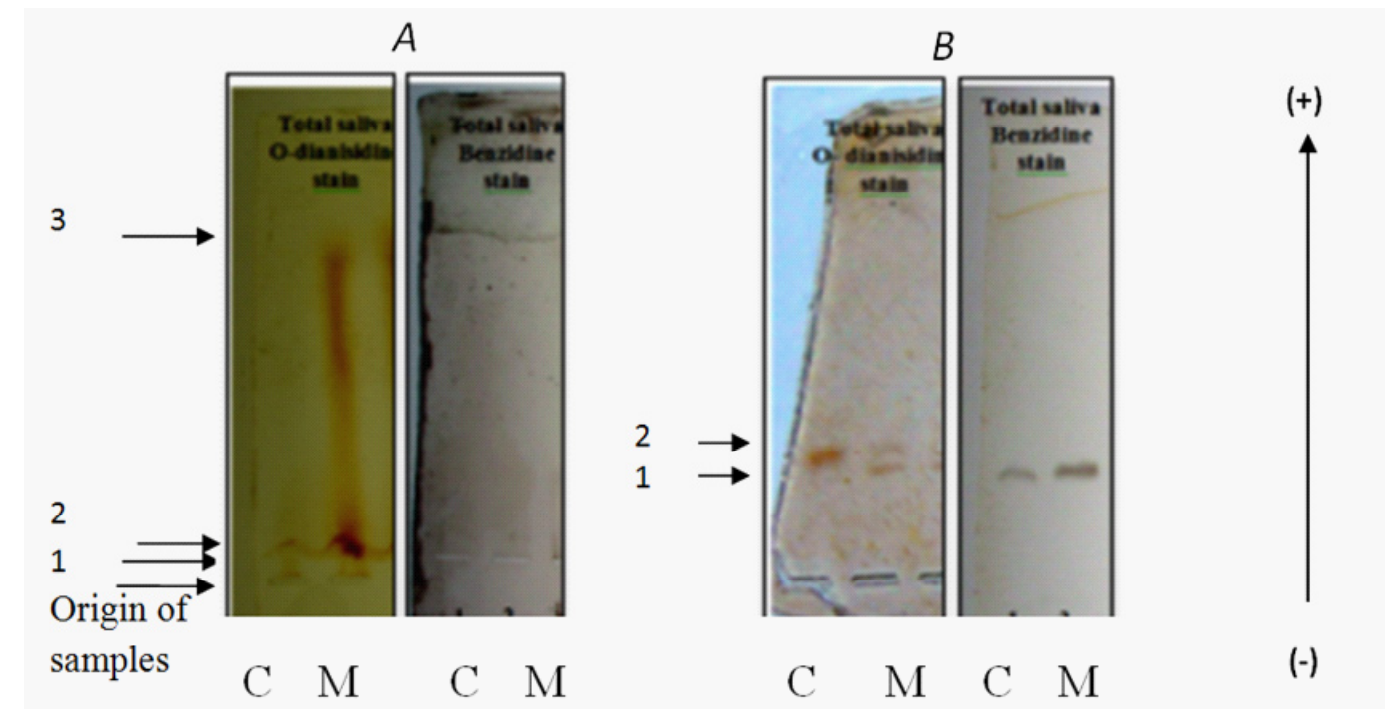

Fig 3: Electrogram of the oral squamous cell carcinoma (OSCC) group patient showing the peroxidase forms profile in the saliva samples using $(A)$ anodic and $(B)$ cathodic conventional PAGE, \&either: O-dianisidine or Benzidine as a substrates in the staining procedure of the gel. The electrophoresis conditions were as described in the material \& methods section. The crude samples that applied were: $(C)$ pooled crude saliva of the control group. (M) pooled crude saliva sample of patients with Malignant tumors (OSCC) 


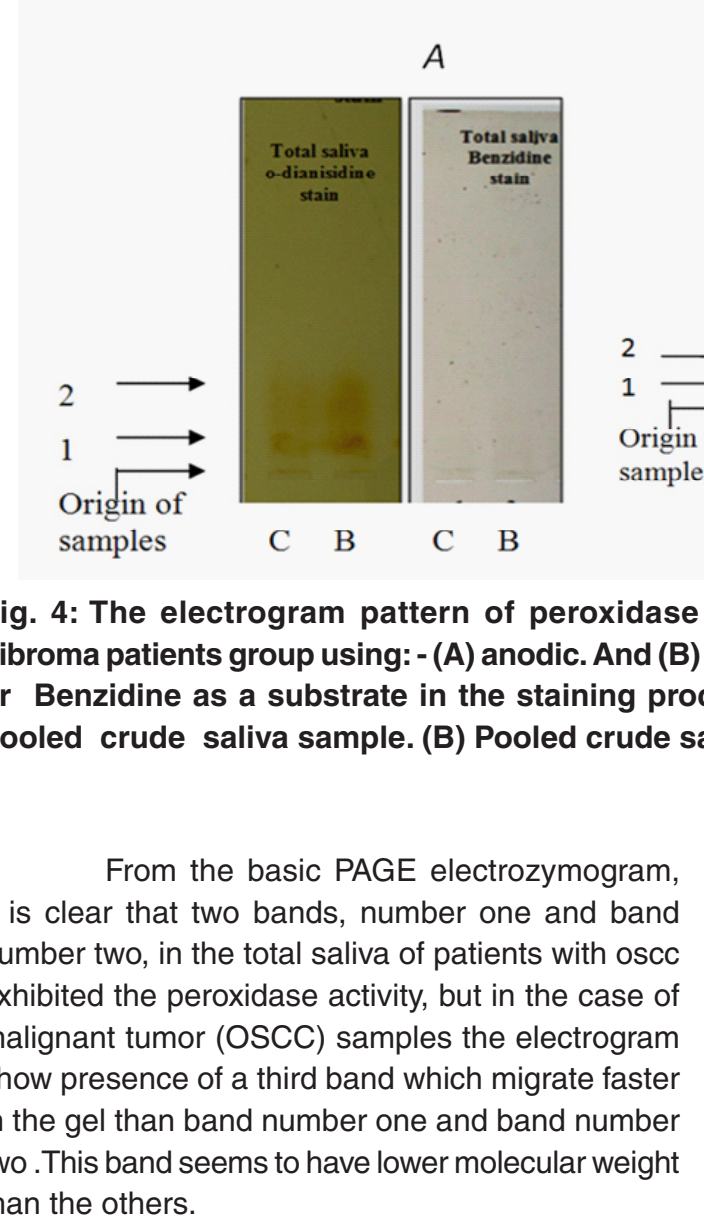

Salivary peroxidase is one of the most important scavenger enzymes of the antioxidant system of submandibular glands acting to prevent the attacks of free radicals \& the appearance of cancer $^{18}$. It protects oral mucosa from cellular lysis induced by hydrogen peroxide, which plays a key role in human cancer development, since it is with the other ROS cause DNA base alteration, strand breaks, damaged tumor suppressor genes and enhanced expression of proto-oncogene ${ }^{19}$. The appearance of the new form of peroxidase ( Figure 3 ,band 3 ), which may be synthesized by the tumor tissue itself, is an attempt to combat the enhanced free radical activity, which causes endothelial damage .It is worth to mention that synthesis of some enzymes of the oxidative system was reported to be induced by free radicals which are being increased at oxidative stress ${ }^{20}$.

\section{REFERENCES}

1. Mestecky J, Lamm M, Strober W, Mcghee J \& Mayer L, Mucosal immunology. Academic press Publications, 80-83 (2005).

2. Russell MW, Bobek LA, Brock JH, Hajishengallis G, Tenovuo J. Mestecky J, Bienenstock J, Lamm ME, Mayer L, Strober W\& Mc Ghee JR, Mucosal Immunology, Academic Press/Elsevier Publications, San Diego, 73-93 (2005).

3. Ihalin R, Loimaranta $V$ \& Tenovuo J, Origin, structure, and biological activities of peroxidases in human saliva. Arch Biochem
Biophys, 445 (2):261-268 (2006).

4. Marcozzi GA rapid procedure for the purification of human salivary peroxidase. Biomed Chromatog, 10: 97-98(1996).

5. Taylor KL, Guzman GS, Pohl J \& Kinkade JM, Distinct chromatographic forms of human hemi - myeloperoxidase obtained by reductive cleavage of the dimeric enzyme. J. Biolo Chem, 265(26): 15938-15946 (1990).

6. Vilja P, Lumikari M, Tenovuo J, Sievers G \& Tuohimaa $P$, Sensitive immunometric assays for secretory peroxidase and myeloperoxidase in human saliva. $J$ Immuno Meth, 141: 277- 
284 (1991).

7. Davies MJ, Hawkins CL, Pattison DI \& Rees MD, Mammalian heme peroxidases: from molecular mechanisms to health implications. Antioxid Redox Signal, 10(7): 1199-1234 (2008).

8. Reznick AZ, Klein I, Eiserich JP, Ctoss CE \& Nagler RM, Inhibition of oral peroxidase activity by cigarette smoke: in vivo and in vitro studies. Free Radic Biol Med, 34(3): 377-384(2003).

9. Hasan $\mathrm{RH}$ and Dawood RM, Study of peroxidase enzyme \& related elements in Serum \& Saliva of Patients with Oral Epithelial Tumors. Asian J Chem, 24(12): 5553-5557 (2012).

10. Amersham Biosciences, Protein Electrophoresis Technical Manual. Handbook, U.S.A. (1999)

11. Azen EA, Salivary peroxidase (SAPX): genetic modification and relationship to the prolinerich $(\mathrm{Pr})$ and acidic $(\mathrm{Pa})$ proteins. Biochem Genet, 15(1): 9-29 (1977).

12. Bakkenist ARJ, Wever R, Vulsma T, Plat H \& Van Gelder BF, Isolation procedure and some properties of myeloperoxidase from human leucocytes. Biochem. Biophys. Acta, 524: 45-54 (1978).

13. Andrews CP \& Krinsky NI, The reductive cleavage of Myeloperoxidase in half, producing enzymically active hemi-myeloperoxidase. J. Biol Chem, 26(49): 4211-4218 (1981).
14. Lee BR, Kim KY, Jung WJ, Avice JC, Ourry A \& Kim TH, Peroxidases and lignification in relation to the intensity of water-deficit stress in white clover (Trifolium repens L). J Exp Bot, 58(6): 1271 - 1279(2007).

15. Thomas PE, Ryan D \& Levin W, An improved staining procedure for the detection of the peroxidase activity of cytochrome P-450 on sodium dodecyl sulfate polyacrylamide gels. Anal. Biochem, 75: 168-176 (1976).

16. Sadasivam S, Manickam A, Biochemical methods, New age international, New Delhi, 145 (2008).

17. Minaguchi k \& Bennick A, Genetics of human salivary proteins. J. Dent Res, 68(1): 2-15 (1989).

18. Filip R, Sebastian T, Ferraro G \& Anesini C, Effect of llex extracts and isolated compounds on peroxidase secretion of rat submandibulary glands. Food and Chem. Toxic. 45, 64965(2007).

19. Bahar G, Feinmasser R,Shpitzer T,Popovtzer $A$,\& Nagler RM. Salivary analysis in oral cancer patients.Cancer.1,109,54-59 (2007)

20. Pruitt M, Kamau D, Miller K, ManssonRahemtulla B, \& Rahemtulla F. Quantitative, standardized assays for determining the concentrations of Bovine lactoperoxidase, human salivary peroxidase, and human myeloper-oxidase. Anal. Biochem. 191(2): 278-86 (1990). 